\title{
Influencia del diámetro de la barra y del recubrimiento en las características adherentes del hormigón armado
}

\author{
M. MOLINA'1, J. P. GUTIÉRREZ1', M. D. GARCÍA² \\ ${ }^{1}$ Instituto de Ciencias de la Construcción Eduardo Torroja (CSIC), 28033 Madrid (España). \\ ${ }^{2}$ Escuela Técnica Superior de Arquitectura (UPM), 28040 Madrid (España).
}

\begin{abstract}
En este artículo se estudia el fenómeno de la adherencia en estructuras de hormigón armado. En primer lugar se incluye una revisión del estado del conocimiento sobre la adherencia y sobre los distintos ensayos de caracterización, principalmente tipo pull-out (ensayos de tracción sobre probetas prismáticas con diferentes longitudes de anclaje según el diámetro de la barra) y ensayos Beam Test sobre vigas de hormigón armado. En segundo lugar se presentan los resultados de los ensayos tipo pull-out realizados en la nave de ensayos del Instituto de Ciencias de la Construcción Eduardo Torroja, en probetas prismáticas variando el diámetro y el recubrimiento de las barras según el ensayo normalizado por la RILEM/CEB/FIP con algunas modificaciones. Se realizan ensayos tanto con la barra en posición centrada como con diferentes recubrimientos. El objeto de los ensayos con excentricidad es estimar la influencia del escaso o nulo recubrimiento en el comportamiento del hormigón armado. Finalmente se analizan los diferentes tipos de fallo obtenidos en los ensayos y la influencia del diámetro de la armadura y el recubrimiento en la transferencia de tensiones hormigón acero.
\end{abstract}

Palabras clave: Adherencia, hormigón armado, ensayo pull-out, ensayo de adherencia en vigas.

\section{Steel/concrete bond characterisation on reinforced concrete structures}

The bond mechanism on reinforced concrete structures is analysing in this paper. A state of art review of bond phenomenon and characterisation tests, pull-out and beam tests are included. Experimental modifications of RILEM/CEB/FIP pull-out tests on prismatic specimens with different concrete cover are also studied. The objective of eccentric tests is to estimate the maximum bond stress and the loss of concrete cover influence on the reinforced concrete behaviour. Bars diameter and concrete cover influence on concrete-steel stress transfer and different types of failures are analysed finally.

Keywords: Bond, Reinforced concrete, tests, pull-out and beam test.

\section{INTRODUCCIÓN}

Con la intención de profundizar en el estudio de la adherencia entre el hormigón y el acero se realiza una revisión del estado del conocimiento sobre los ensayos que la caracterizan, según el ensayo propuesto por la RILEM/CEB/FIP con algunas modificaciones para estudiar su efecto en estructuras de hormigón armado con deficiente recubrimiento.

Con estos ensayos se pretende estudiar la tensión máxima de adherencia y la relación adherencia local-deslizamiento de barras de distinto diámetro y diferente recubrimiento. Los resultados proporcionan una información útil respecto a longitudes de anclaje y solapes, de aplicación práctica en estudio de patologías y reparación de estructuras de hormigón.

\section{ADHERENCIA HORMIGÓN-ACERO}

Han sido numerosas las contribuciones de investigadores a describir el mecanismo de adherencia en el hormigón armado. De entre todas destaca la de Tepfers (1973) (1) que señala que la transferencia de tensiones desde la barra embebida al hormigón se produce mediante unas fuerzas inclinadas de compresión según un ángulo $\alpha$. Equilibrando la componente radial de esta fuerza aparece un anillo de tracciones que provoca fisuraciones internas. Según las condiciones de confinamiento de la barra el fallo se producirá por splitting, fisuración del recubrimiento o pull-out, arrancamiento de la barra.
Además intervienen otros factores como la resistencia del hormigón, tipología de barras, posición de barras durante el hormigonado, recubrimiento y longitud embebida.(2)

\section{ANTECEDENTES ENSAYOS DE CARACTERIZACIÓN DE ADHERENCIA HORMIGÓN-ACERO.}

A partir del ensayo normalizado de adherencia pull-out propuesto por la RILEM/CEB/FIP (2) en el año 1970 se han empleado una gran variedad de ensayos con el objeto de caracterizar las barras de acero y analizar la tensión de adherencia.

A continuación se describen los principales tipos de ensayos de adherencia: el de arrancamiento pull-out y el ensayo de adherencia en vigas beam test.

En los primeros ensayos pull-out prismáticos, Rehm (1961), analizaba el comportamiento de la adherencia local en una única corruga, situaba la longitud adherente en el centro de la barra, apareciendo problemas debido al rozamiento entre la probeta y la placa de apoyo. En el año 1979 Losberg realiza el ensayo con una longitud adherida igual a $3 \varnothing$ obteniendo por el procedimiento de ensayo la tensión máxima de adherencia. Hasta que en los años 70 se normaliza el ensayo pull-out con pequeña longitud adherente, RILEM/CEB/FIP. La región de la barra con adherencia $(l=5 \phi)$ se desplaza respecto al ensayo de Rehm al extremo pasivo de la barra. Para evitar el rozamiento se coloca una plancha de goma entre la superficie de apoyo y la probeta. 
Con el objeto de conocer otros aspectos como la variación de la tensión de adherencia a la que está sometida la barra anclada o solapada, se han realizado ensayos con longitudes adherentes mayores $(1>5 \varnothing)$.

El ensayo de adherencia en vigas Beam-Test es el adoptado tanto por la Instrucción de hormigón española EHE (3) como por el CEB para la homologación de barras corrugadas de acero. Consiste en realizar un ensayo de flexión sobre una viga biapoyada mediante dos cargas puntuales simétricas. De este tipo de ensayo se obtienen igualmente las gráficas tensión de adherencia local/desplazamientos tomando como tensión de adherencia la media entre las correspondientes a los desplazamientos de 0,01- 0,1 y $1 \mathrm{~mm}$.

A partir del ensayo normalizado de la RILEM/CEB/FIP (4) de adherencia pull-out y beam test varios países europeos adoptaron algunos de los métodos propuestos mientras que otros recurrieron a métodos de ensayos diferentes. Este hecho puede presentar inconvenientes debido a que el acero estructural se exporta a distintos países donde la normativa de caracterización de adherencia de barras no es la misma. La disponibilidad de un ensayo normalizado europeo quizás facilitaría el control de calidad del acero y completaría las normativas europeas que todavía no definen un ensayo de este tipo. Actualmente el fib Task Group 4/5(5) se encuentra analizando las posibilidades de un sistema de ensayos de adherencia que proporcionen valores relativos al comportamiento estructural ya que las actuales normativas no lo contemplan, siendo de gran utilidad tanto para fabricantes de acero como para proyectistas de estructuras de hormigón armado.

\section{OBJETO DE LA INVESTIGACIÓN}

El objeto de la investigación experimental es conocer por un lado la influencia del diámetro del refuerzo en la relación tensión de adherencia local/deslizamiento y por otro la influencia del escaso recubrimiento en la pérdida de adherencia. El espesor del recubrimiento juega un papel fundamental en la adherencia ya que mejora la resistencia frente a la fisuración paralela a las armaduras. El parámetro determinante es la relación entre el recubrimiento y el diámetro de la barra.

Los ensayos empleados son tipo pull-out con diferentes recubrimientos según las directrices del CEB sobre probetas prismáticas de hormigón para diferentes diámetros de barra: ø12, ø16 y ø20 mm. Con los ensayos centrados se consigue la máxima resistencia a la adherencia y con los ensayos descentrados la fisuración del recubrimiento.

\section{PROGRAMA EXPERIMENTAL}

Se han desarrollado tres series de ensayos tipo pull-out con distintos recubrimientos. Cada serie contempla un diámetro nominal de barra, la serie 1 corresponde a barras $ø 12 \mathrm{~mm}$, la serie 2 a barras de $\varnothing 16$ mm y por último la serie 3 a barras de ø20 $\mathrm{mm}$ para analizar no sólo la influencia del recubrimiento en la tensión de adherencia local sino la influencia de la relación recubrimiento-diámetro [c/ø] en los resultados, tensión máxima de adherencia, deslizamiento y tipo de fallo.

Las dimensiones de las probetas son $200 \times 200$ × 200 mm, siendo la longitud adherente igual a cinco veces el diámetro de la barra. La falta de adherencia de la barra en la zona de aplicación de la carga, se consigue mediante la colocación de unos "manguitos" de plástico de las mismas características de los que se utilizan en el ensayo normalizado de caracterización de barras de acero, Norma UNE 36740-98. (6)

En cada serie la probeta de hormigón se apoya sobre una plancha de goma de dimensiones $300 \times 300 \times 5 \mathrm{~mm}$ y a su vez sobre una placa de acero de las mismas dimensiones y $10 \mathrm{~mm}$ de espesor para garanti-

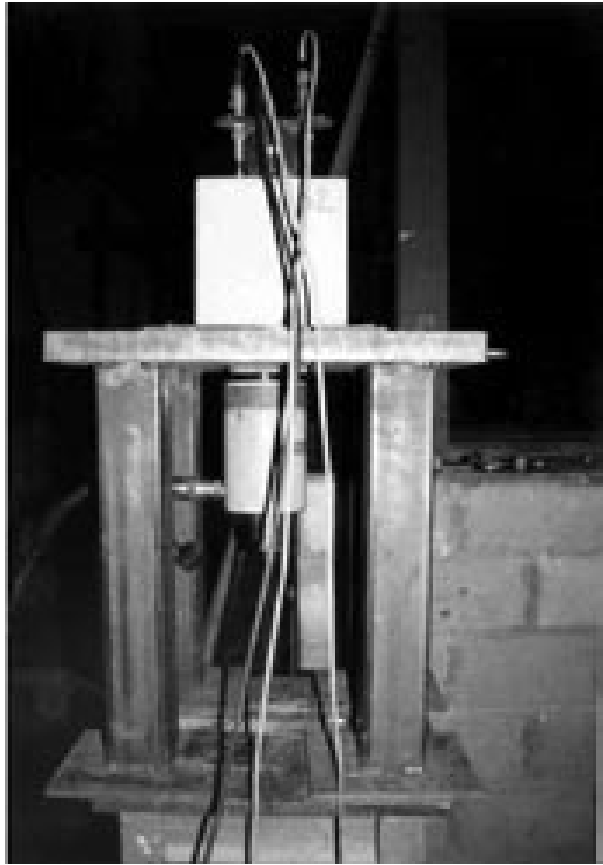

Fig. 1. Ensayo pull-out con captadores de desplazamiento en la parte superior y gato hidráulico en la inferior.

zar un apoyo uniforme y el reparto de tensiones. En la fotografía de la Figura 1 se presenta el dispositivo de ensayo. Consiste en aplicar una carga de tracción creciente mediante un gato hidráulico en el extremo de la barra embebida, registrando el deslizamiento relativo de la barra en el extremo opuesto al de aplicación de la carga.

Este ensayo está basado en las recomendaciones de la RILEM/ CEB / FIB a excepción de que contempla distintas posiciones de la barra en la probeta. A continuación se describe las series ensayadas.

\subsection{Serie 1}

En esta serie se realizan ensayos tipo pull-out con barras del tipo B-400 S y diámetro nominal igual a $12 \mathrm{~mm}$. En la fabricación del hormigón de todas las series se empleó la siguiente dosificación: Cemento tipo I 42,5 - $250 \mathrm{~kg} / \mathrm{m}^{3}$; grava rodada $(4 / 16)-1300 \mathrm{~kg} / \mathrm{m}^{3}$; arena $(0 / 2)$ - $600 \mathrm{~kg} / \mathrm{m}^{3}$; agua - $160 \mathrm{l} / \mathrm{m}^{3}$. La resistencia a compresión a los 28 días del hormigón empleado es 31 MPa y la resistencia a tracción estimada según la instrucción vigente de hormigón estructural EHE (4) es de 2,08 MPa. La longitud adherida de la barra es igual a $60 \mathrm{~mm}$. Las dimensiones de la probeta $200 \times 200 \times 200 \mathrm{~mm}$, permiten obtener una relación recubrimiento/ diámetro de 7,8 .

En esta serie se han contemplado 5 valores nominales de recubrimiento 7,8ø-4ø-2,6ø-2ø-0ø y se han realizado tres ensayos de cada caso realizando un total de 15 ensayos. En la Tabla 1 se recogen los datos de esta serie, donde el número anterior a la letra de cada tipo de ensayo hace referencia al diámetro de la barra.

\subsection{Serie 2}

En esta serie se ensayan probetas prismáticas con barras de diámetro nominal igual a $16 \mathrm{~mm}$ y un hormigón de resistencia a compresión a los 28 días igual a 29,74 MPa. El tipo de acero empleado es el mismo en todas las series. La longitud adherida de la barra es $80 \mathrm{~mm}$ ya que según las recomendaciones del CEB debe ser igual a 5 ø. 
TABla 1. Programa eXPerimental

\begin{tabular}{|c|c|c|c|c|c|}
\hline Tipo & $\begin{array}{c}\text { Recub } \\
\text { Real } \\
c \\
{[\mathbf{m m}]}\end{array}$ & $\begin{array}{r}\text { Relación } \\
\qquad / ø\end{array}$ & Tipo & $\begin{array}{c}\text { Recub. } \\
\text { Real } \\
c \\
{[\mathrm{~mm}]}\end{array}$ & $\begin{array}{r}\text { Relación } \\
c / \varnothing\end{array}$ \\
\hline 12A1 & 94,0 & 7,80 & 16A1 & 92,0 & 5,75 \\
\hline $12 \mathrm{~A} 2$ & 94,0 & 7,80 & $16 \mathrm{~A} 2$ & 92,0 & 5,75 \\
\hline $12 \mathrm{~A} 3$ & * & ${ }^{*}$ & 16B1 & 65,0 & 4,06 \\
\hline 12B1 & 47,0 & 3,90 & 16B2 & 63,2 & 3,95 \\
\hline 12B2 & 47,0 & 3,90 & 16D1 & 37,0 & 2,31 \\
\hline $12 \mathrm{~B} 3$ & 49,2 & 4,10 & $16 \mathrm{D} 2$ & 34,0 & 2,12 \\
\hline $12 \mathrm{C} 1$ & 30,5 & 2,50 & 16E1 & 2,1 & 0,13 \\
\hline $12 \mathrm{C} 2$ & 30,6 & 2,50 & 16E2 & 2,0 & 0,12 \\
\hline $12 \mathrm{C} 3$ & 32,4 & 2,70 & $20 \mathrm{~A} 1$ & 86,0 & 4,30 \\
\hline 12D1 & 22,3 & 1,80 & $20 \mathrm{~A} 2$ & 89,0 & 4,45 \\
\hline $12 \mathrm{D} 2$ & 22,4 & 1,90 & 20B1 & 60,0 & 3,00 \\
\hline 12D3 & 24,2 & 2,00 & 20B2 & 62,0 & 3,10 \\
\hline 12E1 & 5,1 & 0,40 & 20D1 & 37,0 & 1,85 \\
\hline 12E2 & 5,0 & 0,40 & 20D2 & 32,0 & 1,60 \\
\hline 12E3 & 4,0 & 0,30 & 20E1 & 0 & 0 \\
\hline & & & 20E2 & 0 & 0 \\
\hline
\end{tabular}

${ }^{*}$ Los resultados no son representativos, por un fallo de disposición del manguito de plástico.

Debido a que en la serie 1, primera serie realizada en el tiempo, no se obtuvieron datos significativos entre relaciones $c / \varnothing=3$ y $c / \varnothing=4$ para valorar la influencia del recubrimiento en la tensión local de adherencia , en las siguientes series se eliminó la relación recubrimiento diámetro igual a $3 ø$.

Se analizan cuatro valores de recubrimiento, $\mathrm{c}=5,75 \varnothing, 4 \varnothing, 2 \varnothing \mathrm{y}$ 0 , para las probetas tipo A, B, D y E respectivamente realizando dos ensayos en cada caso (Tabla 1 y Figura 2).

\subsection{Serie 3}

El diámetro nominal de la barra de acero ensayada en esta serie es de $20 \mathrm{~mm}$ y la longitud adherida es igual a $100 \mathrm{~mm}$. La resistencia a compresión a los 28 días del hormigón es de 29,74 MPa. Al igual que en la serie anterior se analizan cuatro valores nominales de recubrimiento, $\mathrm{c}=4,5 \varnothing$ (probetas tipo A), $\mathrm{c}=4 \varnothing$ (probetas tipo $\mathrm{B}$ ), $\mathrm{c}=2 \varnothing$ (probetas tipo $\mathrm{D}$ ), y $\mathrm{c}=0$ (probetas tipo $\mathrm{E}$ ), y se realizan dos ensayos en cada caso. Los datos del ensayo se presentan igualmente en la Tabla 1.

\section{RESULTADOS OBTENIDOS}

La tensión media de adherencia local se calcula como la carga aplicada en el ensayo dividida por la superficie adherente de la barra, siendo $\mathrm{Q}$ la carga aplicada [N], ø el diámetro nominal de la barra [mm] y $\mathrm{l}_{\mathrm{b}}$ la longitud adherente $[\mathrm{mm}]$ de acuerdo con la siguiente expresión:

$$
\pi(\mathrm{Mpa})=\frac{\mathrm{Q}}{\mathrm{A}}=\frac{\mathrm{Q}}{\partial \cdot \phi \cdot \mathrm{I}_{\mathrm{b}}}
$$

En la Figura 3 se presenta un diagrama típico tensión de adherencia local $\tau[\mathrm{MPa}]$ en función del deslizamiento del extremo superior de la barra s [mm] obtenido de una de las probetas de la serie 1 con una relación recubrimiento/diámetro igual a 3,9. El fallo producido se origina por el deslizamiento de la barra respecto al hormigón que la rodea

En la Tabla 2 se presentan algunos de los resultados obtenidos de los ensayos realizados, tensión máxima de adherencia en MPa correspondiente a la carga máxima aplicada y deslizamiento relativo de la barra respecto a la superficie de hormigón para esta tensión en mm.

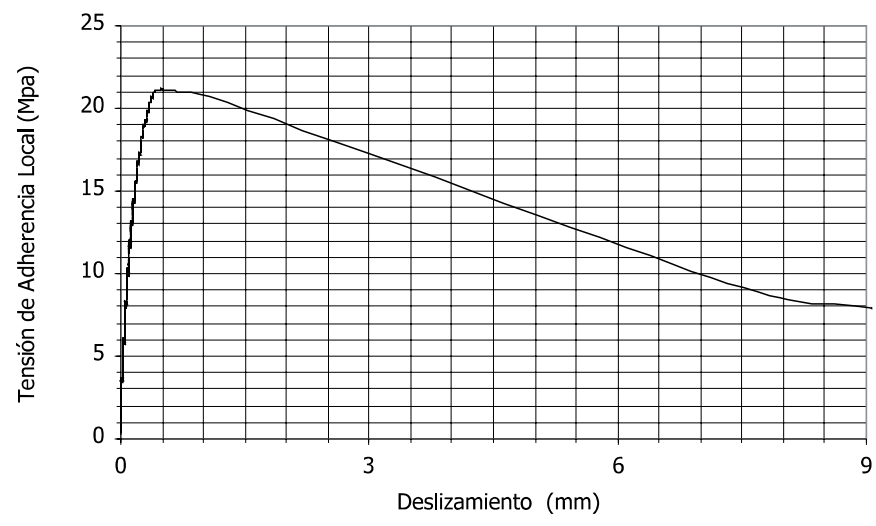

Fig. 3. Gráfica de tensión de adherencia local/ deslizamiento en un ensayo ø $12 \mathrm{~mm}, \mathrm{y} \mathrm{c}=3,9 \varnothing$ (12B2).

\section{SERIE $2 / \varnothing 16$}

Tipo A $\mathrm{c}=92 \mathrm{~mm}[5.75 \varnothing]$

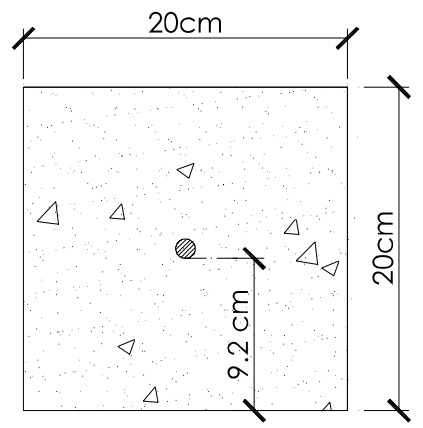

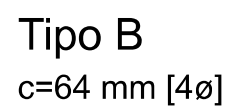

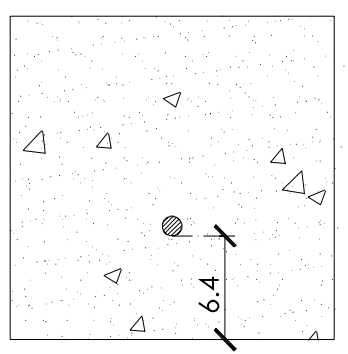

Tipo D $\mathrm{c}=32 \mathrm{~mm}[2 \varnothing]$

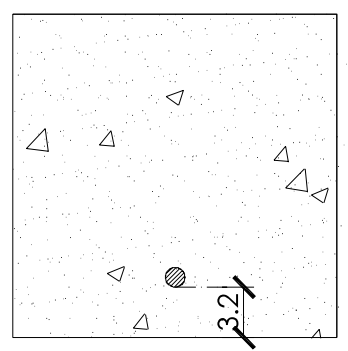

\section{Tipo E $\mathrm{c}=0 \mathrm{~mm}$}

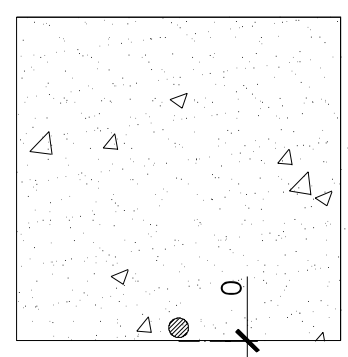

Fig. 2. Probetas Serie 2 con variaciones de recubrimiento. 
TABLA 2. RESULTADOS OBTENIDOS DEL ENSAYO TIPO PULL-OUT

\begin{tabular}{|c|c|c|c|c|c|}
\hline Tipo & $\begin{array}{c}\text { Tensión } \\
\text { Máxima } \\
\text { Adh } \\
\tau_{\text {max }} \\
{[\mathrm{MPa}]}\end{array}$ & $\begin{array}{c}\text { Desliz } \\
\text { para } \tau_{\text {máx }}\end{array}$ & $\begin{array}{c}\mathrm{d} \\
{[\mathrm{mm}]}\end{array}$ & $\begin{array}{c}\text { Tensión } \\
\text { Máxima } \\
\text { Adh } \\
\tau_{\text {máx }} \\
{[\mathrm{MPa}]}\end{array}$ & $\begin{array}{c}\text { Desliz } \\
\text { para } \tau_{\text {máx }} \\
\mathrm{d} \\
{[\mathrm{mm}]}\end{array}$ \\
\hline 12A1 & 18,55 & 1,03 & $16 \mathrm{~A} 1$ & 11,36 & 0,83 \\
\hline 12A2 & 18,96 & 1,17 & $16 \mathrm{~A} 2$ & 10,96 & 0,54 \\
\hline 12A3 & $*$ & $*$ & $16 \mathrm{~B} 1$ & 13,46 & 0,64 \\
\hline 12B1 & 19,77 & 0,33 & $16 \mathrm{~B} 2$ & 15,77 & 0,96 \\
\hline 12B2 & 21,20 & 0,49 & $16 \mathrm{D} 1$ & 13,85 & 0,57 \\
\hline 12B3 & 20,17 & 1,34 & $16 \mathrm{D} 2$ & 16,46 & 0,73 \\
\hline 12C1 & 14,71 & 0,80 & $16 \mathrm{E} 1$ & 4,08 & 0,07 \\
\hline 12C2 & 15,61 & 0,19 & $16 \mathrm{E} 2$ & 5,22 & 0,18 \\
\hline 12C3 & 16,11 & 0,89 & $20 \mathrm{~A} 1$ & 20,44 & 0,74 \\
\hline 12D1 & 13,51 & 0,35 & $20 \mathrm{~A} 2$ & 17,64 & 1,72 \\
\hline 12D2 & 12,37 & 0,55 & $20 \mathrm{~B} 1$ & 19,82 & 0,40 \\
\hline 12D3 & 13,67 & 0,49 & $20 \mathrm{~B} 2$ & 23,99 & 0,12 \\
\hline 12E1 & 10,00 & 0,16 & $20 \mathrm{D} 1$ & 11,90 & 0,05 \\
\hline 12E2 & 8,68 & 0,28 & $20 \mathrm{D} 2$ & 11,66 & 0,17 \\
\hline 12E3 & 9,02 & 0,28 & $20 \mathrm{E} 1$ & 6,77 & 0,08 \\
\hline & & & $20 \mathrm{E} 2$ & 5,55 & 0,12 \\
\hline
\end{tabular}

*Los resultados no son representativos, por un fallo de disposición del manguito de plástico.

En la Figura 4 se presenta la influencia del recubrimiento en la relación tensión de adherencia local/deslizamiento para la serie de ensayos 3 con diámetro nominal de barra igual a $20 \mathrm{~mm}$. Como se observa en la gráfica para un mismo diámetro de armadura la tensión máxima de adherencia aumenta con el recubrimiento debido al efecto de confinamiento pasivo que realiza el hormigón. Únicamente se representan valores pequeños de recubrimiento, hasta $60 \mathrm{~mm}$, debido a que a partir de un cierto valor la tensión local de adherencia se estabiliza. Este valor corresponde al recubrimiento necesario que garantiza el anclaje de barras.

La influencia del diámetro de la barra en la tensión de adherencia local se representa en la Figura 5 para relaciones recubrimiento/ diámetro del orden de $\mathrm{c} / \varnothing=0$ y $\mathrm{c} / \varnothing=2$. Según esta gráfica la tensión última de adherencia local aumenta al disminuir el diámetro. Al aumentar el diámetro nominal de 12 a 16 milímetros la tensión máxima de adherencia en probetas con recubrimientos escasos o nulos, es decir, con una relación recubrimiento diámetro igual a 0 , disminuye aproximadamente un $50 \%$ respecto a la media y para el caso de ø20 $\mathrm{mm}$ disminuye un $33 \%$ respecto al de $\varnothing 12 \mathrm{~mm}$. En el caso de una relación $c / \varnothing=2$ la disminución de la tensión máxima de adherencia para $\varnothing 20 \mathrm{~mm}$ es un $10 \%$ menor que para ø12 mm.

Respecto al tipo de fallo es necesario distinguir que en general para altas relaciones recubrimiento/ diámetro de la barra, por ejemplo en ensayos centrados $12 \mathrm{~A}, 16 \mathrm{~A}$ y $20 \mathrm{~A}$, el fallo se produce por arrancamiento de la barra, pull-out y para aquellos recubrimientos que proporcionan un menor confinamiento, es decir, en probetas con la barra en posición descentrada se origina la fisuración del recubrimiento en la dirección de la barra, fallo que se conoce como splitting (7). Este tipo de fallo es frágil y puede ser originado por ejemplo por un cambio de volumen de una barra debido a corrosión. Sin embargo, conociendo la relación tensión de adherencia local deslizamiento y las condiciones de confinamiento es posible comprobar aunque el recubrimiento se encuentre fisurado si las condiciones del anclaje son o no fiables desde el punto de vista de la seguridad (8).

En la fotografía de la Figura 6 se presenta la fisuración y pérdida del recubrimiento de la probeta de la serie $3,20 \mathrm{E} 2$ con escaso recubrimiento.

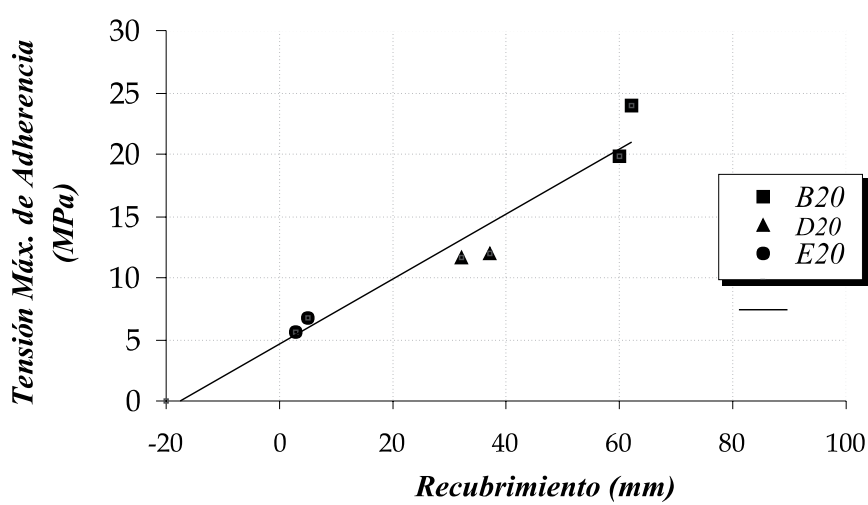

Fig. 4. Influencia del recubrimiento en la tensión máxima de adherencia.

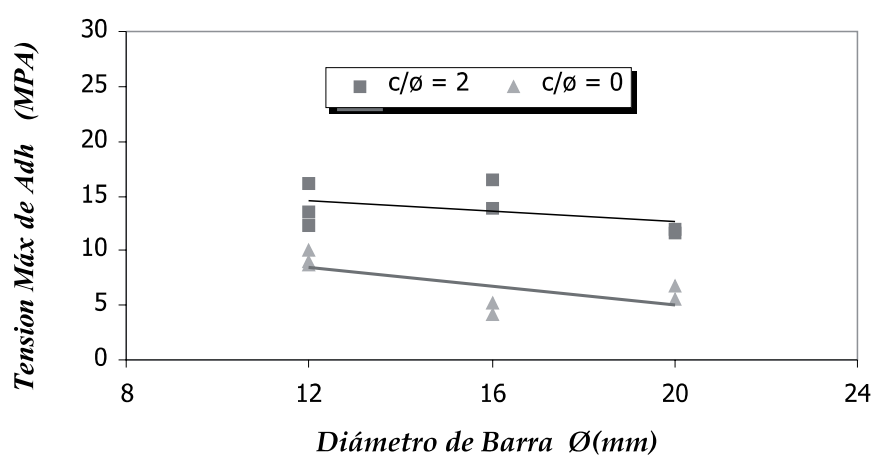

Fig. 5. Influencia del diámetro del refuerzo en la tensión máxima de adherencia.

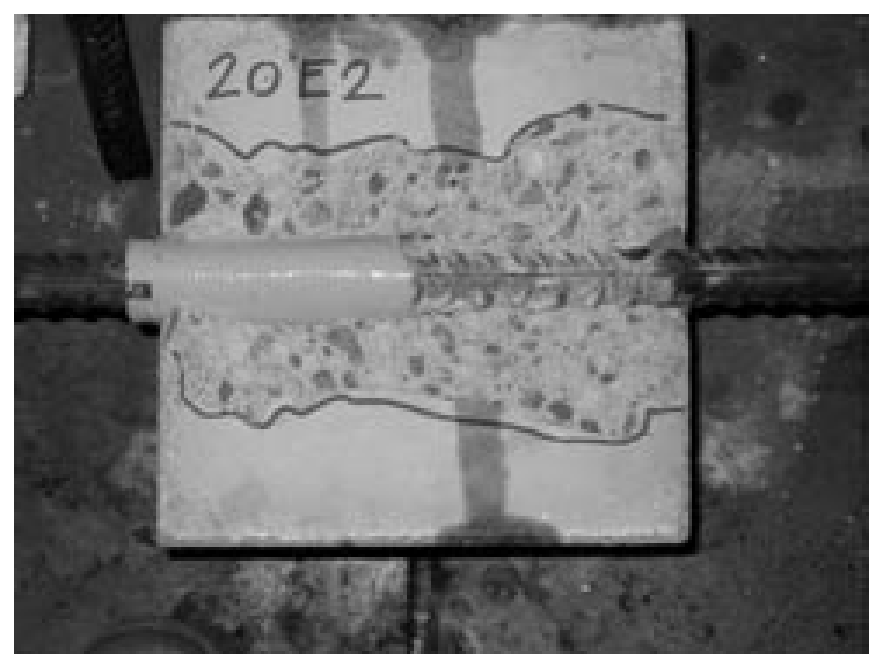

Fig. 6. Desprendimiento del recubrimiento en probeta de la Serie 3 [ø20].

\section{CONCLUSIONES}

El presente artículo recoge los resultados de la investigación experimental sobre el mecanismo de adherencia local entre el hormigón y el acero y a su vez sobre la influencia de algunos parámetros como son el recubrimiento y el diámetro de la armadura.

Entre las conclusiones principales destacan; la tensión máxima de adherencia local aumenta con el recubrimiento hasta una cierta relación recubrimiento diámetro aproximadamente igual a $\mathrm{c} / \varnothing=4$ a partir de la cual se estabiliza ya que queda garantizada la formación del anillo de tracciones en el hormigón que equilibra las fuerzas de adherencia y a partir de este límite es escasa la influencia del recubri- 
miento. En estructuras deterioradas que han perdido el recubrimiento por efecto de ambientes agresivos, corrosión de la armadura o falta de mantenimiento con recubrimientos nulos o escasos, puede decirse según los resultados obtenidos que existe una tensión de adherencia local mínima debido a que en ausencia de recubrimiento el hormigón todavía rodea a la barra en su perímetro quedando una tensión de adherencia mínima que no garantiza en todos los casos el anclaje de las barras. Respecto a la influencia del diámetro de la barra no sólo influye en la tensión de adherencia en el tipo de fallo, ya que para un mismo recubrimiento al aumentar el diámetro de la barra la tendencia al splitting es mayor.

\section{AGRADECIMIENTOS}

Este estudio forma parte del proyecto de investigación PB98-0515 "Análisis de estructuras de hormigón armado con una deficiente transferencia de tensiones hormigón acero" financiado por el Ministerio de Educación y Cultura con cargo al Programa Sectorial de Promoción General del Conocimiento que se ha desarrollado en el Instituto de Ciencias de la Construcción Eduardo Torroja. Los autores agradecen al personal técnico y laboral de la nave de ensayos las facilidades y colaboración prestada a lo largo de la fase experimental.

\section{BIBLIOGRAFÍA}

1. R. Tepfers. «A theory of bond applied to overlapped tensile reinforcement splices for deformed bars». $328 \mathrm{pp}$. Doctoral Thesis. Ed. Chalmers University of Technology. Division of Concrete Structures, Goteborg (Sweden). Publication 73:2. 1973.

2. CEB-FIP. «Código Modelo CEB-FIP 1990 para hormigón estructural». 572 pp. Ed. Colegio de Ingenieros de Caminos, Canales y Puertos; GEHO-CEB Grupo Español del Hormigón; Asociación Técnica Española del Pretensado, Madrid (España), 1996.

3. CEB. «RILEM/CEB/FIP Recommendations on reinforcement steel for reinforced concrete. RC-6: Bond test for reinforcement steel: 2. Pull-out test», Revised Edition, 5 pp, 1983.

4. Comision Permanente del Hormigón. «Instrucción de Hormigón Estructural EHE». 472 pp. Ed. Ministerio de Fomento. Madrid (España) 1998.

5. fib Task Group 4/5 Meeting, Brescia, Mayo 2001.

6. AENOR. «UNE 36740:1998. Determinación de la adherencia de las barras y alambres de acero para armaduras de hormigón armado. Ensayo de la viga», 1998.

7. Fib. «Bulletin $\mathrm{N}^{\mathrm{o}}$ 10: Bond of Reinforcement in Concrete. State-of-Art Report». 427 pp. Ed. International federation for structural concrete (fib). Laussane (Switzerland) 427 pp. 2000.

8. P. G. Gambarova, G. P. Rosati. «Bond and splitting in bar pull-out: behavioural laws». Magazine of Concrete Research 49 [179] 99-110 (1997).

Recibido: 1.2 .03

Aceptado: 30.11 .03 